\title{
Microtubules interacting with a boundary: Mean length and mean first-passage times
}

\author{
Bela M. Mulder \\ FOM Institute AMOLF, Science Park 104, 1098XG Amsterdam, the Netherlands
}

(Received 17 April 2012; published 2 July 2012)

\begin{abstract}
I formulate a dynamical model for microtubules interacting with a catastrophe-inducing boundary. In this model microtubules are either waiting to be nucleated, actively growing or shrinking, or stalled at the boundary. I first determine the steady-state occupation of these various states and the resultant length distribution. Next, I formulate the problem of the mean first-passage time to reach the boundary in terms of an appropriate set of splitting probabilities and conditional mean first-passage times and solve explicitly for these quantities using a differential equation approach. As an application, I revisit a recently proposed search-and-capture model for the interaction between microtubules and target chromosomes [M. Gopalakrishnan and B. S. Govindan, Bull. Math. Biol. 73, 2483 (2011)]. I show how my approach leads to a direct and compact solution of this problem.
\end{abstract}

DOI: 10.1103/PhysRevE.86.011902

PACS number(s): 87.17.Aa, 87.16.Ka, 87.10.Ca

\section{INTRODUCTION}

Microtubules (hereafter abbreviated as MTs) are filamentous macropolymers built from tubulin dimers. They are one of the components of the cytoskeleton of all eukaryotic cells. They play a number of roles, including providing mechanical stability to the cell, serving as transport pathways enabling linear transport of versicular cargo by motor proteins, and providing forces for the positioning of organelles and other intracellular components (for a general overview see Ref. [1]). Perhaps their most striking function appears during mitosis wherein they form the mitotic spindle, the machinery for positioning and separating the duplicated chromosomes prior to cell division. They owe their functional plasticity to an intrinsic so-called "dynamical instability" mechanism [2] that causes individual MTs to stochastically alternate between growing and shrinking states. By controlling this dynamical process, through MT associated proteins (MAPs) that nucleate new MTs or selectively stabilize or destabilize them by locally or globally changing the rates with which they switch between dynamical states, cells are able to reconfigure MT assemblies on time scales as fast as a few minutes. The canonical model to describe MT dynamics was developed in the early 1990s by Dogterom and Leibler [3]. This model showed that isolated MTs, depending on their dynamical parameters, can either be in a regime of bounded growth leading to an exponential length distribution in a steady state or in a regime of unbounded growth in which the MT length on average increases linearly in time. Of course, MTs "live" within the confines of a finite size cell, whose dimensions $(\sim 10 \mu \mathrm{m})$ are comparable to the observed lengths of MTs. Interactions between MTs and boundaries, be it the cell cortex or the surface of other intracellular compartments, are therefore important. Indeed, a number of MT functions depends critically on these interactions: examples are nuclear positioning in yeast [4], spindle positioning in C. elegans [5], and the orientation of the cortical MT array in plant cells [6].

In spite of this clear relevance, it appears that a systematic approach to the theory of MTs interacting with boundaries is lacking in the literature. The one problem of this type which did receive substantial attention is the search-and-capture mechanism by which MTs are thought to find the condensed chromosomes prior to mitosis [7-10], involving estimating the mean first-passage time of a MT to hit a limited size target at a distance from its nucleation point. However, although these works in fact do contain some of the basic features of the MT-boundary problem, it is mostly hidden (literally in the case of Ref. [8], actually an unpublished thesis) under the specifics of the intended application. Moreover, these works also rely heavily on "forward" techniques involving the time evolution of the full probability density for reaching a given state from specified initial conditions. Although this approach obviously yields a full solution of the problem, for passagetime problems, which effectively require integrating over final states, the full probability density is in a sense a form of "overkill". The treatment of this type of problems can in fact be simplified considerably by using "backward" techniques as is, e.g., elegantly illustrated for diffusion problems in Redner's monograph [11]. In the present work I show how this approach can be used from the ground up to solve the problem of a MT interacting with a boundary.

The outline of the paper is as follows. In Sec. II, I introduce the dynamical model of a MT interacting with a boundary; solve for its steady-state properties, such as the average length; and choose an appropriate set of nondimensionalized parameters and variables. In Sec. III, I turn to the analysis of the mean first-passage time to the boundary, formally solving this in terms of a small set of splitting probabilities and conditioned mean first-passage times, which are subsequently determined explicitly through the solution of appropriate linear boundary value problems. I then use some biological data on MT dynamics to estimate the order of magnitudes for the quantities involved. Finally, in Sec. IV, I revisit the recent search-and-capture model discussed by Gopalakrishnan and Govindan [10] and show how it is compactly solved using the techniques introduced. I then finish with a number of concluding remarks and two technical appendices.

\section{MEAN LENGTH}

\section{A. Dynamical model}

The standard two-state dynamical instability model describes MTs with length $l$ that are either growing with velocity $v_{+}$or shrinking with velocity $v_{-}$and can switch between the growing and the shrinking states (a catastrophe) with a 


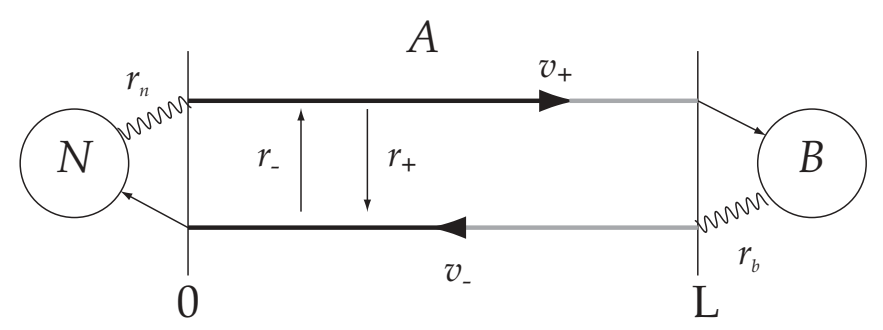

FIG. 1. Schematic of our model showing the three components of the state space: the nucleation state $N$ from which MTs are nucleated at rate $r_{n}$, the active state $A$ in which MTs either grow or shrink with velocities $v_{+}$and $v_{-}$and switch between these two states with rates $r_{+}$and $r_{-}$, and a barrier state $B$ from which MTs exit at a rate $r_{b}$.

constant rate $r_{+}$and between the shrinking and the growing states (a rescue) with a constant rate $r_{-}$. Collectively, I call these two states the active states and denote the corresponding state space by $A$. I extend this model by two more states: a nucleation state $N$ in which a MT enters upon shrinking back to zero length and from which it can be (re)nucleated into a zero-length growing state at a constant rate $r_{n}$ and a boundary state $B$ in which a MT enters upon hitting a boundary at a distance $L$ from the nucleation point and which it leaves in a shrinking state at a rate $r_{b}$. Formally, the state space of this extended model is therefore given by $\Omega=N \cup A \cup B$. I illustrate the model and its state space in Fig. 1.

It should be noted that from a biophysical point of view, the model is of course an idealization. In reality a growing MT impinging on a boundary will generate forces. These forces will affect the growth velocity and the propensity to switch to the shrinking state so that the latter is no longer a simple Poisson process [12]. Moreover, these forces may also deform the boundary, lead to buckling of the MT, or cause it to slide along the boundary (for a review see Ref. [13]). These additional complexities, however, will have limited impact on the results to be presented here as long as the residence time at the boundary is small compared to the time to traverse the distance between nucleation point and boundary, which is certainly the case for effectively reflective boundaries for which $r_{b} \gg r_{+}$, and I choose to ignore them here.

The dynamics of the model described above defines a time-homogeneous Markov process on the full state space. It is, however, technically convenient to split the dynamics on the "active" part of the state space $A$ from those on the two "waiting" states $N$ and $B$ and deal with the communication between these different states through boundary conditions. I, therefore, first define the probability densities (per unit length) $m_{s}\left(l, t \mid \omega_{0}, t_{0}\right)$ for an active MT to have length $l$ and be in state $s=+,-$ at time $t$, given that it was in some state $\omega_{0}$ at time $t_{0}<t$. These densities satisfy the evolution equations

$$
\begin{aligned}
\frac{\partial}{\partial t} m_{+}\left(l, t \mid \omega_{0}, t_{0}\right)= & -v_{+} \frac{\partial}{\partial l} m_{+}\left(l, t \mid \omega_{0}, t_{0}\right)-r_{+} m_{+}\left(l, t \mid \omega_{0}, t_{0}\right) \\
& +r_{-} m_{-}\left(l, t \mid \omega_{0}, t_{0}\right), \\
\frac{\partial}{\partial t} m_{-}\left(l, t \mid \omega_{0}, t_{0}\right)= & v_{-} \frac{\partial}{\partial l} m_{-}\left(l, t \mid \omega_{0}, t_{0}\right)-r_{-} m_{-}\left(l, t \mid \omega_{0}, t_{0}\right) \\
& +r_{+} m_{+}\left(l, t \mid \omega_{0}, t_{0}\right) .
\end{aligned}
$$

Likewise, I define the probability $M_{n}\left(t \mid \omega_{0}, t_{0}\right)$ for the MT to be in the nucleation state at time $t$, given that it was in some state $\omega_{0} \in \Omega$ at time $t_{0}<t$. This probability satisfies the evolution equation

$$
\frac{\partial}{\partial t} M_{n}\left(t \mid \omega_{0}, t_{0}\right)=v_{-} m_{-}\left(0, t \mid \omega_{0}, t_{0}\right)-r_{n} M_{n}\left(t \mid \omega_{0}, t_{0}\right)
$$

The probability $M_{b}\left(t \mid \omega_{0}, t_{0}\right)$ for the MT to be in the boundary state at time $t$ in turn satisfies

$$
\frac{\partial}{\partial t} M_{b}\left(t \mid \omega_{0}, t_{0}\right)=v_{+} m_{+}\left(L, t \mid \omega_{0}, t_{0}\right)-r_{b} M_{b}\left(t \mid \omega_{0}, t_{0}\right) .
$$

This system of equations is closed by the boundary conditions

$$
\begin{aligned}
& v_{+} m_{+}\left(0, t \mid \omega_{0}, t_{0}\right)=r_{n} M_{n}\left(t \mid \omega_{0}, t_{0}\right), \\
& v_{-} m_{-}\left(L, t \mid \omega_{0}, t_{0}\right)=r_{b} M_{b}\left(t \mid \omega_{0}, t_{0}\right) .
\end{aligned}
$$

By construction, the dynamics on the full state space conserves probability, and indeed if I define the total probability

$$
\begin{aligned}
M\left(t \mid \omega_{0}, t_{0}\right)= & M_{n}\left(t \mid \omega_{0}, t_{0}\right)+M_{a}\left(t \mid \omega_{0}, t_{0}\right)+M_{b}\left(t \mid \omega_{0}, t_{0}\right) \\
= & M_{n}\left(t \mid \omega_{0}, t_{0}\right)+\int_{0}^{\infty} d l\left\{m_{+}\left(l, t \mid \omega_{0}, t_{0}\right)\right. \\
& \left.+m_{-}\left(l, t \mid \omega_{0}, t_{0}\right)\right\}+M_{b}\left(t \mid \omega_{0}, t_{0}\right),
\end{aligned}
$$

then

$$
\frac{\partial}{\partial t} M\left(t \mid \omega_{0}, t_{0}\right)=0,
$$

allowing me to set $M\left(t \mid \omega_{0}, t_{0}\right)=1$.

\section{B. Steady-state behavior}

In the steady state the probabilities do not depend on time nor on initial conditions, allowing me to write the evolution equations as a set of balance equations

$$
\begin{gathered}
v_{+} \frac{d}{d l} m_{+}(l)=-r_{+} m_{+}(l)+r_{-} m_{-}(l), \\
-v_{-} \frac{d}{d l} m_{-}(l)=-r_{-} m_{-}(l)+r_{+} m_{+}(l), \\
v_{-} m_{-}(0)=r_{n} M_{n}, \\
v_{+} m_{+}(L)=r_{b} M_{b},
\end{gathered}
$$

to be supplemented by the boundary conditions

$$
\begin{aligned}
& v_{+} m_{+}(0)=r_{n} M_{n}, \\
& v_{-} m_{-}(L)=r_{b} M_{b} .
\end{aligned}
$$

Adding Eqs. (9) and (10) yields

$$
\frac{d}{d l}\left\{v_{+} m_{+}(l)-v_{-} m_{-}(l)\right\}=0 .
$$

Combining either Eqs. (11) and (13) or Eqs. (12) and (14) shows that the constant of integration vanishes, and hence,

$$
v_{+} m_{+}(l)=v_{-} m_{-}(l) .
$$

This identity is now used to eliminate $m_{-}(l)$ from Eq. (9) from which I then readily find that for $l \leqslant L$,

$$
m_{+}(l)=\frac{r_{n} M_{n}}{v_{+}} e^{-l / \bar{l}},
$$




$$
m_{-}(l)=\frac{r_{n} M_{n}}{v_{-}} e^{-l / \bar{l}}
$$

where the length

$$
\bar{l}=\left(\frac{r_{+}}{v_{+}}-\frac{r_{-}}{v_{-}}\right)^{-1}
$$

is of course only positive when $r_{+} v_{-}-r_{-} v_{+}>0$, the socalled bounded-growth regime and represents the steady-state average length of an active MT in the absence of the boundary. Although in the presence of boundaries one can also consider the unbounded-growth regime $r_{+} v_{-}-r_{-} v_{+}<0$ as was done, e.g., in Ref. [14] for the case wherein the boundary is fully reflecting, I will not do so here and focus exclusively on the bounded-growth case. The dependence of the probability that the MT is in the boundary state can, from Eq. (12), be shown to obey the following relationship:

$$
M_{b}=\frac{r_{n}}{r_{b}} e^{-\frac{L}{l}} M_{n} .
$$

The normalization condition (7) can then be used to determine the probabilities for a MT to be in a nucleation state, an active state, and a boundary state, respectively,

$$
\begin{aligned}
& M_{n}=\frac{1}{1+r_{n} \bar{l}\left(\frac{1}{v_{+}}+\frac{1}{v_{-}}\right)\left(1-e^{-L / \bar{l}}\right)+\frac{r_{n}}{r_{b}} e^{-L / \bar{l}}}, \\
& M_{a}=\frac{r_{n} \bar{l}\left(\frac{1}{v_{+}}+\frac{1}{v_{-}}\right)\left(1-e^{-L / \bar{l}}\right)}{1+r_{n} \bar{l}\left(\frac{1}{v_{+}}+\frac{1}{v_{-}}\right)\left(1-e^{-L / \bar{l}}\right)+\frac{r_{n}}{r_{b}} e^{-L / \bar{l}}}, \\
& M_{b}=\frac{\frac{r_{n}}{r_{b}} e^{-L / \bar{l}}}{1+r_{n} \bar{l}\left(\frac{1}{v_{+}}+\frac{1}{v_{-}}\right)\left(1-e^{-L / \bar{l}}\right)+\frac{r_{n}}{r_{b}} e^{-L / \bar{l}}} .
\end{aligned}
$$

Taking the limit $L \rightarrow \infty$, I find $M_{a} \propto r_{n} \bar{l}\left(\frac{1}{v_{+}}+\frac{1}{v_{-}}\right)$. As density $\propto$ nucleation rate $\times$ lifetime, this suggests that the time

$$
\bar{t}=\bar{l}\left(\frac{1}{v_{+}}+\frac{1}{v_{-}}\right)=\frac{v_{+}+v_{-}}{r_{+} v_{-}-r_{-} v_{+}}
$$

is the expected lifetime of an, otherwise unconstrained, zerolength newly nucleated MT, a result indeed first derived by Rubin [15].

I now define the mean length of the active MTs

$$
\langle l\rangle_{a}=\frac{1}{M_{a}} \int_{0}^{L} d l l\left\{m_{+}(l)+m_{-}(l)\right\}=\bar{l} \frac{\left[1-\left(1+\frac{L}{l}\right) e^{-L / \bar{l}}\right]}{\left(1-e^{-L / \bar{l}}\right)} .
$$

The time-averaged length of all MTs present is then simply

$$
\langle l\rangle=\frac{M_{a}}{M_{a}+M_{b}}\langle l\rangle_{a}+\frac{M_{b}}{M_{a}+M_{b}} L .
$$

I can readily check the limits $\langle l\rangle_{a} \rightarrow \bar{l}$ when $L \rightarrow \infty$ and $\langle l\rangle_{a} \simeq \frac{1}{2} L$ for $L \rightarrow 0$. The latter limit can be understood by considering that for very small $L$, the growing and shrinking traversal times $L / v_{+}$and $L / v_{-}$become small with respect to the mean time between catastrophes $1 / r_{+}$and rescues $1 / r_{-}$, respectively, so that the MT is deterministically "bouncing" between the end points $l=0$ and $l=L$.

\section{Dimensional analysis}

I have deliberately deferred the dimensional analysis of the system up to this point to allow the results of the steady-state solution to guide me to a natural choice of the units of length and time. In view of Eqs. (21) and (25), I choose $\bar{l}$ of Eq. (24) as the unit length and $\bar{t}$ as the unit of time. For completeness sake, I can also introduce the unit of speed

$$
\frac{1}{\bar{v}} \equiv \frac{\bar{t}}{\bar{l}}=\frac{1}{v_{+}}+\frac{1}{v_{-}} .
$$

By convention I will adopt the Greek alphabet to denote dimensionless quantities. I introduce the dimensionless parameters: growth speed $v_{+}=v_{+} / \bar{v}$, shrinkage speed $v_{-}=v_{-} / \bar{v}$, catastrophe rate $\rho_{+}=r_{+} \bar{t}$, rescue rate $\rho_{-}=r_{-} \bar{t}$, nucleation rate $\rho_{n}=r_{n} \bar{t}$, barrier unbinding rate $\rho_{b}=r_{b} \bar{t}$, and distance to barrier $\Lambda=L / \bar{l}$. Note that these assignments, which have the clear advantage of maximizing the interpretability of the nondimensional equations, do have the disadvantage of leaving dependencies among the parameters as by construction

$$
\begin{aligned}
& \frac{1}{v_{+}}+\frac{1}{v_{-}}=1, \\
& \frac{\rho_{+}}{v_{+}}-\frac{\rho_{-}}{v_{-}}=1 .
\end{aligned}
$$

To denote the independent variables of time and length, I write $\tau$ and $\lambda$, respectively. Finally, the densities, as my dependent variables, are denoted by $\mu_{s}\left(\lambda, \tau \mid \omega_{0}, \tau_{0}\right)=\bar{l} m_{s}\left(\lambda \bar{l}, \tau \bar{t} \mid \omega_{0}, \tau_{0} \bar{t}\right)$.

Using these notations, the steady-state results of the previous section can be summarized as

$$
\begin{gathered}
M_{n}=\frac{1}{1+\rho_{n}\left(1-e^{-\Lambda}\right)+\frac{\rho_{n}}{\rho_{b}} e^{-\Lambda}}, \\
\langle\lambda\rangle_{a}=\frac{\left[1-(1+\Lambda) e^{-\Lambda}\right]}{\left(1-e^{-\Lambda}\right)} .
\end{gathered}
$$

For future reference I will also rewrite the evolution equations in a more compact notation. To do so, I treat pairs of functions $\left(\varphi_{+}(\lambda, \tau), \varphi_{-}(\lambda, \tau)\right)$, defined on the growing and shrinking parts of the state space, respectively, as a single vector-valued function $\varphi_{s}(\lambda, \tau), s=+,-$. This allows me to write

$$
\frac{\partial}{\partial \tau} \mu_{s}\left(\lambda, \tau \mid \omega_{0}, \tau_{0}\right)=\sum_{s^{\prime}} G_{s, s^{\prime}}^{*}\left[\mu_{s^{\prime}}\left(\lambda, \tau \mid \omega_{0}, \tau_{0}\right)\right],
$$

where $G^{*}$ is the operator matrix

$$
G_{s, s^{\prime}}^{*}=\left(\begin{array}{cc}
-v_{+} \frac{\partial}{\partial \lambda}-\rho_{+} & \rho_{-} \\
\rho_{+} & v_{-} \frac{\partial}{\partial \lambda}-\rho_{-}
\end{array}\right) .
$$

The fact that I use the notation $G^{*}$, signifying the Hermitian conjugate of the generator $G$ of the Markov process, is conventional when discussing the forward Kolmogorov equation, which is the formal term for the evolution equation for the probability densities [16].

\section{THE MEAN-FIRST PASSAGE TIME}

I approach the problem of calculating the mean-first passage time (MFPT) for a microtubule to hit the boundary at a distance $\Lambda$ in three steps. I first provide a formal solution to the problem in terms of a suitably chosen set of survival (and ruin) probabilities. I then calculate the static splitting probabilities that describe the relative weights of the direct and indirect paths of reaching the boundary and finally determine the conditional MFPTs corresponding to these sets of paths. 


\section{A. Formal solution}

I first define the survival set, the subset of state space excluding the boundary state, which I denote by $\Omega_{\Lambda} \equiv \Omega / B=$ $N \cup A$. My goal is to determine the survival probability of the process in this set when starting at $\tau=0$ from an arbitrary active state with length $\lambda<\Lambda$, which I denote by $S_{\Omega_{\Lambda}}(\tau \mid \lambda, s)$. I start by observing that in order for the process to survive in the active state, it should not exit either at length $\Lambda$ into the barrier state $B$ or at length zero into the nucleation state $N$. Therefore, the survival probability in the active state, $S_{A}(\tau \mid \lambda, s)$, can be written as

$$
S_{A}(\tau \mid \lambda, s)=1-R_{A}^{B}(\tau \mid \lambda, s)-R_{A}^{N}(\tau \mid \lambda, s),
$$

which introduces the conditional ruin probabilities $R_{A}^{B}(\tau \mid \lambda, s)$ and $R_{A}^{N}(\tau \mid \lambda, s)$, being the probabilities to have exited into the boundary state at $\lambda=\Lambda$ or the nucleation state at $\lambda=0$ at time $\tau$, respectively, without leaving the active state at any prior moment. Associated to any ruin probability, $R_{O}^{D}(\tau \mid \omega)$ is a corresponding propensity function $\sigma_{O}^{D}(\tau \mid \omega)$, which is the timedependent rate at which the process exits from its "origin" state $O$ into the "destination" state $D$. This propensity is defined as $\sigma_{O}^{D}(\tau \mid \omega)=\frac{\partial}{\partial \tau} R_{O}^{D}(\tau \mid \omega)$, or, equivalently, given a conditional survival probability $S_{O}^{D}(\tau \mid \omega), \sigma_{O}^{D}(\tau \mid \omega)=-\frac{\partial}{\partial \tau} S_{O}^{D}(\tau \mid \omega)$. Using this definition, I can state the following identity:

$$
S_{\Omega_{\Lambda}}(\tau \mid \lambda, s)=S_{A}(\tau \mid \lambda, s)+\int_{0}^{\tau} d \tau^{\prime} \sigma_{A}^{N}\left(\tau^{\prime} \mid \lambda, s\right) S_{\Omega_{\Lambda}}\left(\tau-\tau^{\prime} \mid N\right),
$$

which captures the fact that starting from an active state, the MT survives either by remaining active and not reaching $\Lambda$ or by shrinking back to zero at some intermediate time $\tau^{\prime}$ with rate $\sigma_{A}^{N}\left(\tau^{\prime} \mid \lambda, s\right)$ and then surviving from the nucleation state with probability $S_{\Omega_{\Lambda}}\left(\tau-\tau^{\prime} \mid N\right)$. In the nucleation state a MT survives either by remaining in this state or being nucleated into a growing one at an intermediate time and then surviving from the zero-length growing state, leading to the identity

$$
S_{\Omega_{\Lambda}}(\tau \mid N)=S_{N}(\tau \mid N)+\int_{0}^{\tau} d \tau^{\prime} \sigma_{N}\left(\tau^{\prime} \mid N\right) S_{\Omega_{\Lambda}}\left(\tau-\tau^{\prime} \mid 0,+\right),
$$

where the nucleation propensity is simply given by $\sigma_{N}(\tau \mid N)=$ $\rho_{n} \exp \left(-\rho_{n} \tau\right)$ as nucleation is a Poisson process. As is clear from the steady-state solution, a MT will always leave the active state for a large enough time $\left(M_{n}>0\right.$ independent of the initial conditions) so that $S_{A}(\infty \mid \lambda, s)=0$. The ultimate conditional ruin probabilities $R_{A}^{B}(\infty \mid \lambda, s)$ and $R_{A}^{N}(\infty \mid \lambda, s)$ are usually, and aptly, called splitting probabilities as the total ruin probability is "split" between them: $R_{A}^{B}(\infty \mid \lambda, s)+$ $R_{A}^{N}(\infty \mid \lambda, s)=1$. Using the latter relation, I can rewrite identity (35) as

$$
\begin{aligned}
S_{\Omega_{\Lambda}}(\tau \mid \lambda, s)= & {\left[R_{A}^{B}(\infty \mid \lambda, s)-R_{A}^{B}(\tau \mid \lambda, s)\right]+\left[R_{A}^{N}(\infty \mid \lambda, s)\right.} \\
& \left.-R_{A}^{N}(\tau \mid \lambda, s)\right]+\int_{0}^{\tau} d \tau^{\prime} \sigma_{A}^{N}\left(\tau^{\prime} \mid \lambda, s\right) S_{\Omega_{\Lambda}} \\
& \times\left(\tau-\tau^{\prime} \mid N\right) .
\end{aligned}
$$

I can now define the quantity I am after, viz., the MFPT for the process to pass length $\Lambda$, starting from the active state $(\lambda, s)$ as the integral over the latter survival probability

$$
T_{\Omega_{\Lambda}}(\lambda, s) \equiv \int_{0}^{\infty} d \tau \tau \sigma_{\Omega_{\Lambda}}(\tau \mid \lambda, s)=\int_{0}^{\infty} d \tau S_{\Omega_{\Lambda}}(\tau \mid \lambda, s) .
$$

Considering the integration of the first term on the right-hand side of Eq. (37), I find

$$
\begin{aligned}
& \int_{0}^{\infty} d \tau\left[R_{A}^{B}(\infty \mid \lambda, s)-R_{A}^{B}(\tau \mid \lambda, s)\right] \\
& \quad=\int_{0}^{\infty} d \tau \tau \sigma_{A}^{B}(\tau \mid \lambda, s) \\
& =R_{A}^{B}(\infty \mid \lambda, s) \frac{\int_{0}^{\infty} d \tau \tau \sigma_{A}^{B}(\tau \mid \lambda, s)}{\int_{0}^{\infty} d \tau \sigma_{A}^{B}(\tau \mid \lambda, s)} \\
& \equiv R_{A}^{B}(\infty \mid \lambda, s) T_{A}^{B}(\lambda, s),
\end{aligned}
$$

which introduces the conditional MFPT $T_{A}^{B}(\lambda, s)$ of the process to exit at $\Lambda$ without ever shrinking to zero and where I have used the fact that, by definition, the ruin probability is the time integral over the propensity, i.e.,

$$
R_{A}^{B}(\infty \mid \lambda, s)=\int_{0}^{\infty} d \tau \sigma_{A}^{B}(\tau \mid \lambda, s) .
$$

Similarly, integrating the second term yields

$$
\int_{0}^{\infty} d \tau\left[R_{A}^{N}(\infty \mid \lambda, s)-R_{A}^{N}(\tau \mid \lambda, s)\right]=R_{A}^{N}(\infty \mid \lambda, s) T_{A}^{N}(\lambda, s),
$$

where $T_{A}^{N}(\lambda, s)$ is the MFPT to exit into the nucleation state by shrinking to length zero without first reaching the boundary at $\lambda=\Lambda$. Finally, integrating the last term gives

$$
\begin{aligned}
\int_{0}^{\infty} & d \tau \int_{0}^{\tau} d \tau^{\prime} \sigma_{A}^{N}\left(\tau^{\prime} \mid \lambda, s\right) S_{\Omega_{\Lambda}}\left(\tau-\tau^{\prime} \mid N\right) \\
& =\int_{0}^{\infty} d \tau^{\prime} \sigma_{A}^{N}\left(\tau^{\prime} \mid \lambda, s\right) \int_{\tau^{\prime}}^{\infty} d \tau S_{\Omega_{\Lambda}}\left(\tau-\tau^{\prime} \mid N\right) \\
& =R_{A}^{N}(\infty \mid \lambda, s) T_{\Omega_{\Lambda}}(N) .
\end{aligned}
$$

The MFPT starting $T_{\Omega_{\Lambda}}(N)$ from the nucleation state is readily obtained from Eq. (36) and yields

$$
T_{\Omega_{\Lambda}}(N)=T_{N}(N)+T_{\Omega_{\Lambda}}(0,+)=\frac{1}{\rho_{n}}+T_{\Omega_{\Lambda}}(0,+),
$$

where I have used that exiting the nucleation state is sure, i.e., $S_{N}(\infty \mid N)=0$.

Collecting all these results then yields

$$
\begin{aligned}
T_{\Omega_{\Lambda}}(\lambda, s)= & R_{A}^{B}(\infty \mid \lambda, s) T_{A}^{B}(\lambda, s) \\
& +R_{A}^{N}(\infty \mid \lambda, s)\left\{T_{A}^{N}(\lambda, s)+\frac{1}{\rho_{n}}+T_{\Omega_{\Lambda}}(0,+)\right\} .
\end{aligned}
$$

The interpretation of this result is clear. Starting from $(\lambda, s)$, the MT either exits directly at $\lambda=\Lambda$, which happens with probability $R_{A}^{B}(\infty \mid \lambda, s)$ and (on average) takes a time $T_{A}^{B}(\lambda, s)$, or the MT first shrinks back to $\lambda=0$ [with probability $\left.R_{A}^{N}(\infty \mid \lambda, s)\right]$, which takes a time $T_{A}^{N}(\lambda, s)$, and then has to wait a time $\frac{1}{\rho_{n}}$ to be renucleated after which it takes a time 


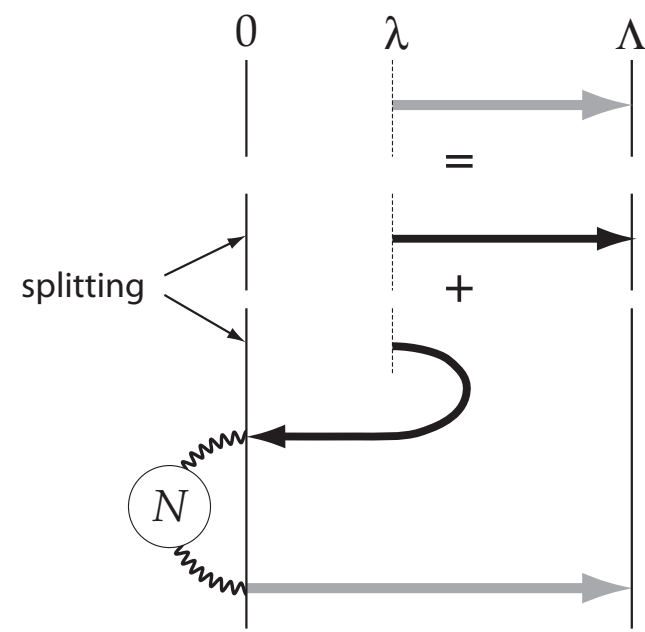

FIG. 2. Schematic showing how the unconditional MFPT (gray arrow) for a growing MT reaches the boundary from starting length $\lambda$ splits into two conditional MFPTs: the first associated with all direct paths (black arrows) from $\lambda$ to the boundary at $\Lambda$ and the second associated with all paths that shrink to zero length without reaching the boundary, spend time in the nucleation state (wiggly line), and then try again unconditionally starting from length zero.

$T_{\Omega_{\Lambda}}(0,+)$ to reach $\lambda=\Lambda$ for the first time. I illustrate this result schematically in Fig. 2.
If I now consider a MT starting at $\lambda=0$ and in the growing state $s=+$, I can self-consistently solve for the MFPT $T_{\Omega_{\Lambda}}(0,+)$, which is my main result:

$$
T_{\Omega_{\Lambda}}(0,+)=T_{A}^{B}(0,+)+\frac{R_{A}^{N}(\infty \mid 0,+)}{R_{A}^{B}(\infty \mid 0,+)}\left\{T_{A}^{N}(0,+)+\frac{1}{\rho_{n}}\right\} .
$$

This latter result is in fact sufficient to solve the general problem because in order to reach the boundary at $\Lambda$ from length zero, the MT first has to pass through each intermediate length $\lambda<\Lambda$, taking time $T_{\Omega_{\lambda}}(0,+)$ (note the subscript $\lambda$ to $\Omega$ here), and then reach $\Lambda$ from there, i.e.,

$$
T_{\Omega_{\Lambda}}(0,+)=T_{\Omega_{\lambda}}(0,+)+T_{\Omega_{\Lambda}}(\lambda,+),
$$

showing that the MFPT for a MT starting in the growing state at an arbitrary length can be expressed fully in terms of MFPTs starting from the zero-length state.

Although due to the fundamental asymmetry of the problem, there is no corresponding simple rule for the shrinking case, the following argument shows how I can leverage the results of the growing case to obtain a fairly compact representation. I first introduce the survival probability of the shrinking state with respect to rescues, which is simply given by $S_{-}(\tau \mid-)=\exp \left(-\rho_{-} \tau\right)$. If no rescue occurs, the shrinking MT will hit zero length at the deterministic time $\tau_{-}(\lambda)=\lambda / \nu_{-}$ so that

$$
\begin{aligned}
S_{\Omega_{\Lambda}}(\tau \mid \lambda,-)= & \left\{1-H\left(\tau-\tau_{-}(\lambda)\right)\right\}\left\{S_{-}(\tau \mid-)+\int_{0}^{\tau} d \tau^{\prime} \sigma_{-}\left(\tau^{\prime} \mid-\right) S_{\Omega_{\Lambda}}\left(\tau-\tau^{\prime} \mid \lambda-\nu_{-} \tau^{\prime},+\right)\right\} \\
& +H\left(\tau-\tau_{-}(\lambda)\right)\left\{\int_{0}^{\tau_{-}(\lambda)} d \tau^{\prime} \sigma_{-}\left(\tau^{\prime} \mid-\right) S_{\Omega_{\Lambda}}\left(\tau-\tau^{\prime} \mid \lambda-\nu_{-} \tau^{\prime},+\right)+S_{-}\left(\tau_{-}(\lambda) \mid-\right) S_{\Omega_{\Lambda}}\left(\tau-\tau_{-}(\lambda) \mid N\right)\right\},
\end{aligned}
$$

where $H(x)$ is the standard Heavyside function. Integrating over all time yields the desired result

$$
\begin{aligned}
T_{\Omega_{\Lambda}}(\lambda,-) & =\frac{1}{\rho_{-}}\left(1-e^{-\rho_{-} \tau_{-}(\lambda)}\right)+\rho_{-} \int_{0}^{\tau_{-}(\lambda)} d \tau e^{-\rho_{-} \tau} T_{\Omega_{\Lambda}}\left(\lambda-v_{-} \tau,+\right)+e^{-\rho_{-} \tau_{-}(\lambda)} T_{\Omega_{\Lambda}}(N) \\
& =\left(1-e^{-\rho_{-} \tau_{-}(\lambda)}\right)\left\{\frac{1}{\rho_{-}}+\frac{\int_{0}^{\tau_{-}(\lambda)} d \tau \sigma_{-}(\tau \mid-) T_{\Omega_{\Lambda}}\left(\lambda-v_{-} \tau,+\right)}{\int_{0}^{\tau_{-}(\lambda)} d \tau \sigma_{-}(\tau \mid-)}\right\}+e^{-\rho_{-} \tau_{-}(\lambda)} T_{\Omega_{\Lambda}}(N) \\
& =R_{-}\left(\tau_{-}(\lambda) \mid-\right)\left\{\frac{1}{\rho_{-}}+\left\langle\left. T_{\Omega_{\Lambda}}\left(\lambda-v_{-} \tau,+\right)\right|_{(\lambda,-)}\right\}+S_{-}\left(\tau_{-}(\lambda) \mid-\right)\left\{\frac{1}{\rho_{n}}+T_{\Omega_{\Lambda}}(0,+)\right\},\right.
\end{aligned}
$$

where $\left\langle T_{\Omega_{L}}\left(\lambda-\nu_{-} \tau,+\right)\right\rangle_{(\lambda,-)}$ is the average MFPT of a MT that starts in a growing state after a single rescue from a shrinking state originally at length $\lambda$ at time zero, provided this happens before the shrinking state hits zero length.

\section{B. The splitting probabilities}

To calculate the splitting probabilities $R_{A}^{N}(\infty \mid \lambda, s)$ (exit at $\lambda=0$ ) and $R_{A}^{B}(\infty \mid \lambda, s)$ (exit at $\left.\lambda=\Lambda\right)$, I first recall from the theory of Markov processes that expectation values of future events seen as functions of the initial time and state satisfy the backward Kolmogorov equation [16]. Specifically, any ruin probability $R_{K}^{C}(T \mid \tau, \lambda, s)$, where $K$ is some subset of $\Omega$ and $C$ is a conditioning event, satisfies

$$
\begin{aligned}
\frac{\partial}{\partial \tau} R_{K}^{C}(T \mid \tau, \lambda, s) & =-\frac{\partial}{\partial T} R_{K}^{C}(T \mid \tau, \lambda, s) \\
& =-\sum_{s^{\prime}} G_{s, s^{\prime}}\left[R_{K}^{C}\left(T \mid \tau, \lambda, s^{\prime}\right)\right],
\end{aligned}
$$

where the generator $G_{s, s^{\prime}}$ is the Hermitian conjugate of the operator (33), i.e.,

$$
G_{s, s^{\prime}}=\left(\begin{array}{cc}
v_{+} \frac{\partial}{\partial \lambda}-\rho_{+} & \rho_{+} \\
\rho_{-} & -v_{-} \frac{\partial}{\partial \lambda}-\rho_{-}
\end{array}\right) .
$$

Since my process is time homogeneous, I can of course take the initial time to be $\tau=0$. Letting my final time $T \rightarrow \infty$, I see 
that $\frac{\partial}{\partial \tau} R_{K}^{C}(\infty \mid \tau, \lambda, s)=\frac{\partial}{\partial \tau} R_{K}^{C}(\infty \mid 0, \lambda, s)=0$ so that splitting probabilities satisfy

$$
\sum_{s^{\prime}} G_{s, s^{\prime}}\left[R_{K}^{C}\left(\infty \mid \lambda, s^{\prime}\right)\right]=0
$$

and are said to be harmonic in analogy with diffusion where the operator $G$ is the Laplacian.

For convenience sake I now drop the explicit mention of the final time and set $R_{A}^{N}(\lambda, s)=R_{A}^{N}(\infty \mid \lambda, s)$ and $R_{A}^{B}(\lambda, s)=$ $R_{A}^{B}(\infty \mid \lambda, s)$. I first consider the splitting probabilities for exiting into the boundary state and write out Eq. (49) to obtain

$$
\begin{array}{r}
v_{+} \frac{\partial}{\partial \lambda} R_{A}^{B}(\lambda,+)-\rho_{+} R_{A}^{B}(\lambda,+)+\rho_{+} R_{A}^{B}(\lambda,-)=0, \\
-v_{-} \frac{\partial}{\partial \lambda} R_{A}^{B}(\lambda,-)-\rho_{-} R_{A}^{B}(\lambda,-)+\rho_{-} R_{A}^{B}(\lambda,+)=0,
\end{array}
$$

with the obvious boundary conditions of surely exiting when in the growing state at the boundary, $R_{A}^{B}(\Lambda,+)=1$, and never exiting at $B$ when starting from the shrinking state at $\lambda=$ $0, R_{A}^{B}(0,-)=0$. I find the following solutions:

$$
\begin{gathered}
R_{A}^{B}(\lambda,+)=\frac{e^{\lambda}-\left(1-\lambda_{+}\right)}{e^{\Lambda}-\left(1-\lambda_{+}\right)}, \\
R_{A}^{B}(\lambda,-)=\frac{\left(e^{\lambda}-1\right)\left(1-\lambda_{+}\right)}{e^{\Lambda}-\left(1-\lambda_{+}\right)},
\end{gathered}
$$

where I have introduced the mean forward run length of a MT, $\lambda_{+}=v_{+} / \rho_{+}$. In an analogous manner, the splitting probabilities for shrinking back to zero length, $R_{A}^{N}(\lambda, s)$, are also readily determined to be

$$
\begin{gathered}
R_{A}^{N}(\lambda,+)=\frac{e^{\Lambda}-e^{\lambda}}{e^{\Lambda}-\left(1-\lambda_{+}\right)}, \\
R_{A}^{N}(\lambda,-)=\frac{e^{\Lambda}-\left(1-\lambda_{+}\right) e^{\lambda}}{e^{\Lambda}-\left(1-\lambda_{+}\right)} .
\end{gathered}
$$

One checks that these forms satisfy the a priori requirements $R_{A}^{B}(\lambda,+)+R_{A}^{N}(\lambda,+)=R_{A}^{B}(\lambda,-)+R_{A}^{N}(\lambda,-)=$ 1 , which follow from the fact that the ultimate ruin of a MT on a finite length interval is sure. These splitting probabilities were also derived in Ref. [8] by considering Laplace transforms of recurrence relations satisfied by the probability density.

\section{The conditional MFPTs}

With the splitting probabilities determined, I can directly calculate the conditional MFPTs by solving a time-integrated form of the backward equation. Indeed, integrating Eq. (48) over the final time $T$ and recalling that $R_{K}^{C}(\tau \mid \tau, \lambda, s)=0$ yields as a first step

$$
\begin{aligned}
& -\int_{\tau}^{\infty} d T \frac{\partial}{\partial T} R_{K}^{C}(T \mid \tau, \lambda, s) \\
& =-R_{K}^{C}(\infty \mid \lambda, s) \\
& \quad=-\int_{\tau}^{\infty} d T \sum_{s^{\prime}} G_{s, s^{\prime}}\left[R_{K}^{C}\left(T \mid \tau, \lambda, s^{\prime}\right)\right] .
\end{aligned}
$$

At this point I would like to interchange the integration and the operation of $G_{s, s^{\prime}}$, but as $R_{K}^{C}\left(T \mid \tau, \lambda, s^{\prime}\right)$ tends to a constant for $T \rightarrow \infty$, this is not directly possible. However, I can use the fact that the splitting probabilities are harmonic, i.e., $G_{s, s^{\prime}}\left[R_{K}^{C}\left(\infty \mid \lambda, s^{\prime}\right)\right]=0$, and the linearity $G_{s, s^{\prime}}$ to obtain the identity

$$
\begin{aligned}
& -G_{s, s^{\prime}}\left[R_{K}^{C}\left(T \mid \tau, \lambda, s^{\prime}\right)\right] \\
& \quad=G_{s, s^{\prime}}\left[R_{K}^{C}\left(\infty \mid \lambda, s^{\prime}\right)\right]-R_{K}^{C}\left(T \mid \tau, \lambda, s^{\prime}\right) .
\end{aligned}
$$

Substitution of this identity into Eq. (57) yields an integrable argument exactly of the form previously encountered in Eq. (39) so that

$$
\begin{aligned}
& -\int_{\tau}^{\infty} d T \sum_{s^{\prime}} G_{s, s^{\prime}}\left[R_{K}^{C}\left(T \mid \tau, \lambda, s^{\prime}\right)\right] \\
& =\int_{\tau}^{\infty} d T \sum_{s^{\prime}} G_{s, s^{\prime}}\left[R_{K}^{C}\left(\infty \mid \lambda, s^{\prime}\right)-R_{K}^{C}\left(T \mid \tau, \lambda, s^{\prime}\right)\right] \\
& =\sum_{s^{\prime}} G_{s, s^{\prime}}\left[\int_{\tau}^{\infty} d T\left\{R_{K}^{C}\left(\infty \mid \lambda, s^{\prime}\right)-R_{K}^{C}\left(T \mid \tau, \lambda, s^{\prime}\right)\right\}\right] \\
& =\sum_{s^{\prime}} G_{s, s^{\prime}}\left[R_{K}^{C}\left(\infty \mid \lambda, s^{\prime}\right) T_{K}^{C}\left(\lambda, s^{\prime}\right)\right] .
\end{aligned}
$$

Combining Eqs. (57) and (59) yields the sought-after relation

$$
\sum_{s^{\prime}} G_{s, s^{\prime}}\left[R_{K}^{C}\left(\infty \mid \lambda, s^{\prime}\right) T_{K}^{C}\left(\lambda, s^{\prime}\right)\right]=-R_{K}^{C}(\infty \mid \lambda, s),
$$

which together with appropriate boundary conditions yields a closed form equation for the conditional MFPTs $T_{K}^{C}\left(\lambda, s^{\prime}\right)$. Note that this is in fact a rather general result, valid for all mixed discrete and/or continuous time-homogeneous Markov processes.

I now apply Eq. (60) to my specific problem; starting with the case of exiting at $\Lambda$ into the boundary state $B$, I have

$$
\begin{aligned}
\lambda_{+} & \frac{\partial}{\partial \lambda} R_{A}^{B}(\lambda,+) T_{A}^{B}(\lambda,+)-R_{A}^{B}(\lambda,+) T_{A}^{B}(\lambda,+) \\
& +R_{A}^{B}(\lambda,-) T_{A}^{B}(\lambda,-)=-\frac{1}{\rho_{+}} R_{A}^{B}(\lambda,+) \\
& -\lambda_{-} \frac{\partial}{\partial \lambda} R_{A}^{B}(\lambda,-) T_{A}^{B}(\lambda,-)-R_{A}^{B}(\lambda,-) T_{A}^{B}(\lambda,-) \\
& +R_{A}^{B}(\lambda,+) T_{A}^{B}(\lambda,+)=-\frac{1}{\rho_{-}} R_{A}^{B}(\lambda,-),
\end{aligned}
$$

where I have introduced the mean backward run length, $\lambda_{-}=\nu_{-} / \rho_{-}$. This equation is to be solved with the boundary conditions $R_{A}^{B}(\Lambda,+) T_{A}^{B}(\Lambda,+)=0$ and $R_{A}^{B}(0,-) T_{A}^{B}(0,-)=$ 0 , the former due to the fact that a growing MT at $\lambda=\Lambda$ will immediately exit into the boundary state $B$ while the latter follows as the probability for a shrinking MT starting at $\lambda=0$ to reach the boundary vanishes. Once again the equations for the corresponding quantities for exiting at zero length are entirely similar. In practice I reduce these sets of first-order equations by substitution into inhomogeneous linear second-order equations. These are readily solved by standard techniques, and I present the resultant, rather unwieldy, expressions in Appendix A. As an explicit check on these results, I consider the time it takes a growing MT to shrink back to the origin when the boundary is made irrelevant 
TABLE I. Table of MT dynamical parameters for yeast and interphase plant cells.

\begin{tabular}{lcc}
\hline \hline Parameter & Yeast & Plant \\
\hline Growth speed $(\mu \mathrm{m} / \mathrm{min})$ & 2.4 & 4.8 \\
Shrinking speed $(\mu \mathrm{m} / \mathrm{min})$ & 9.6 & 9.6 \\
Catastrophe rate $(/ \mathrm{min})$ & 0.3 & 0.28 \\
Rescue rate $(/ \mathrm{min})$ & & 0.42 \\
Nucleation rate $(/ \mathrm{min})$ & 0.15 & $0.15^{\text {a }}$ \\
Mean length $($ no boundary $)(\mu \mathrm{m})$ & 8 & 68.5 \\
Expected lifetime (no boundary) $(\mathrm{min})$ & 4.17 & 21.43 \\
\hline \hline
\end{tabular}

${ }^{\mathrm{a}}$ In the absence of available data, we take this number equal to that of yeast.

by placing it at infinity. In that case, the limit of Eq. (A6) becomes

$$
\lim _{\Lambda \rightarrow \infty} T_{A}^{N}(\lambda,+) \equiv T(\lambda,+)=1+\lambda \frac{\left(\rho_{+}+\rho_{-}\right)}{\left(\rho_{+} \nu_{-}-\rho_{-} \nu_{+}\right)} .
$$

Upon redimensionalizing, this expression is identical to the one derived earlier by Bicout from the full time and spacedependent survival probability [17]. As an aside, I note that the result $T(0,+)=1$ shows that the time scale I have adopted is indeed that of the origin return time of an unconstrained MT as already stated in Sec. II B.

\section{Application to biological data}

In order to get a feel for what the results derived above mean in real-world terms, I apply them to two sets of fairly well characterized kinetic parameters for MTs, one derived from observations on fission yeast [18] and one on interphase Tobacco Bright Yellow-2 plant culture cells [19]. These data sets are summarized in Table I.

I now confront these two types of MTs with boundaries located at $5 \mu \mathrm{m}$, smaller than both mean lengths in the absence of boundaries and comparable to half the length of a fission yeast cell; $20 \mu \mathrm{m}$, double the mean length for the yeast MT and still significantly smaller than that of the Tobacco BY-2 MTs; and $100 \mu \mathrm{m}$, on the order of the typical length scale of a Tobacco BY-2 cell. I first consider the splitting probabilities $R_{A}^{B}(l,+)$ and $R_{A}^{N}(l,+)$, which I plot in Fig. 3. I see that for the smallest boundary distance $L=5 \mu \mathrm{m}$ both for yeast and plant MTs, the probability to reach the boundary from zero length is already appreciable and increases roughly linearly with the starting length, consistent with it being dominated by uninterrupted growth. As I increase the boundary distance, the probabilities depend more strongly nonlinearly on the starting length. This is most striking for the case of yeast at $L=$ $100 \mu \mathrm{m}$ at which $R_{A}^{B}(l,+)$ is essentially zero until the starting length is within the natural length $\bar{l} \approx 8 \mu \mathrm{m}$ from the boundary.

Next, I turn to the conditional MFPTs $T_{A}^{B}(l,+)$ and $T_{A}^{N}(l,+)$. Here, I first need to take a little care as for yeast the rescue probability vanishes $\left(r_{-}=0\right)$ so that the backward run length $l_{-}$is ill-defined. One can of course go through the procedure in Sec. III C again, setting $\rho_{-}=0$ at the outset in Eq. (49). However, in this case the conditional MFPTs are also readily determined from first principles:
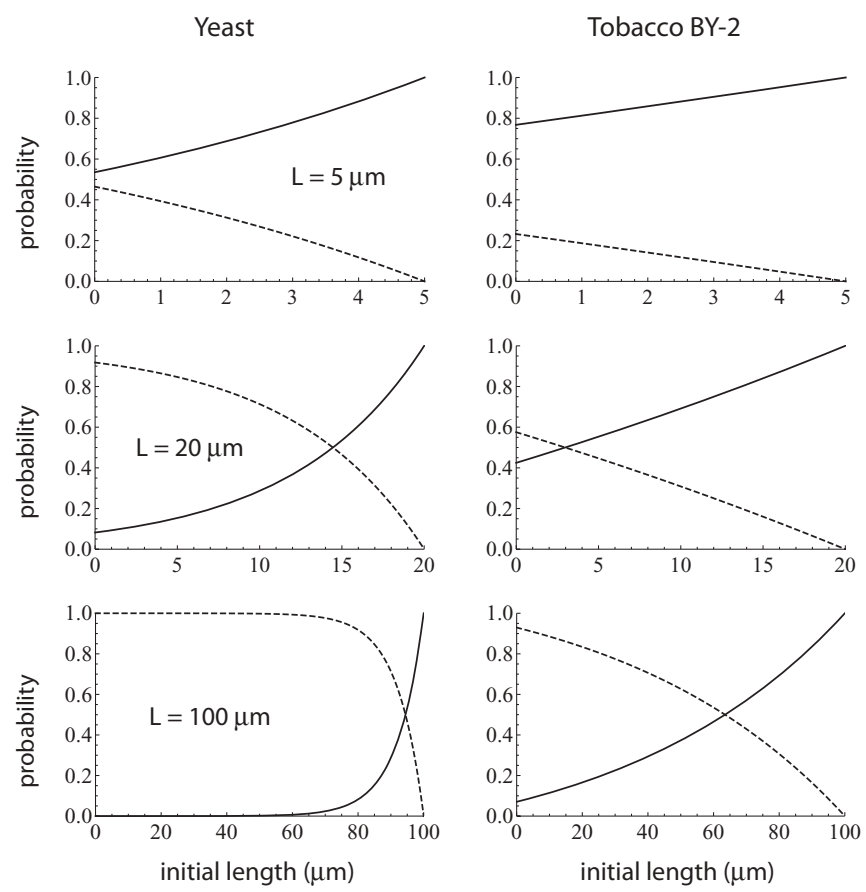

FIG. 3. Splitting probabilities for hitting the barrier, $R_{A}^{B}(l,+)$ (solid line), and shrinking to zero length, $R_{A}^{N}(l,+)$ (dashed line), as a function of the initial length $l$ for different values of the distance to the barrier $L$.

$$
\begin{gathered}
r_{-}=0: T_{A}^{B}(l,+)=\frac{(L-l)}{v_{+}}, \\
r_{-}=0: T_{A}^{N}(l,+)=\frac{\int_{0}^{\frac{(L-l)}{v_{+}}} d t e^{-r_{+} t}\left\{t+\frac{\left(l+v_{+} t\right)}{v_{-}}\right\}}{\int_{0}^{\frac{(L-l)}{v_{+}}} d t e^{-r_{+} t}} \\
=\frac{l}{v_{-}}+\bar{t} \frac{\left[1-e^{-\frac{L-l}{l}}\left(1+\frac{L-l}{\bar{l}}\right)\right]}{1-e^{-\frac{L-l}{l}}},
\end{gathered}
$$

where Eq. (64) follows because a nonrescuable MT can only reach the boundary without first shrinking away by growing towards it deterministically and Eq. (65) is obtained by averaging (i) the time to experience a catastrophe before reaching the boundary plus (ii) the time to shrink to zero length from that moment on over the ensemble of histories that do not reach the boundary. These two approaches indeed give the same results, serving as another independent check on the general formalism. Figure 4 shows the resulting passage times. Perhaps at first sight, a puzzling feature of these results is the decrease of $T_{A}^{N}(l,+)$ for increasing the starting length $l$, which is evident for the yeast case. This, however, is a direct consequence of the conditioning on shrinking back without reaching the boundary. If the starting length is within the forward run length $l_{+}$from the boundary, a conditioned MT must rapidly undergo a catastrophe after which it deterministically shrinks back to zero length. The conditioned return time [the second term on the far right-hand side of Eq. (65)] is therefore a strongly nonlinearly decreasing function of the distance to the boundary whereas the time to deterministically shrink back from the starting length $l / v_{-}$ only increases linearly with length. 
Yeast
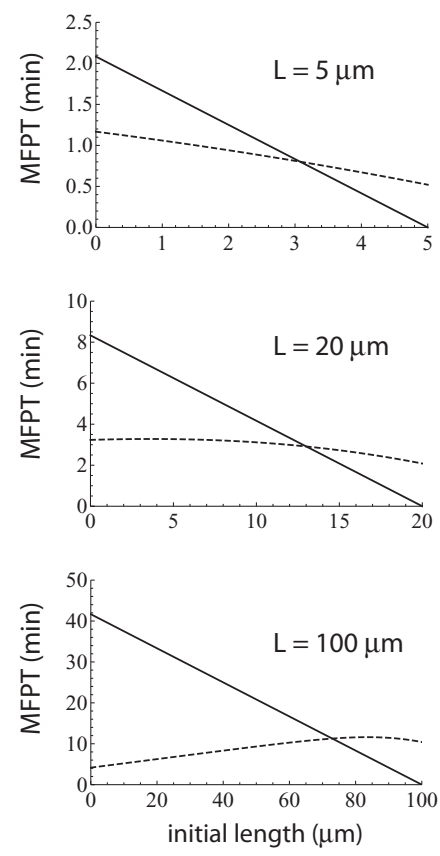

Tobacco BY-2
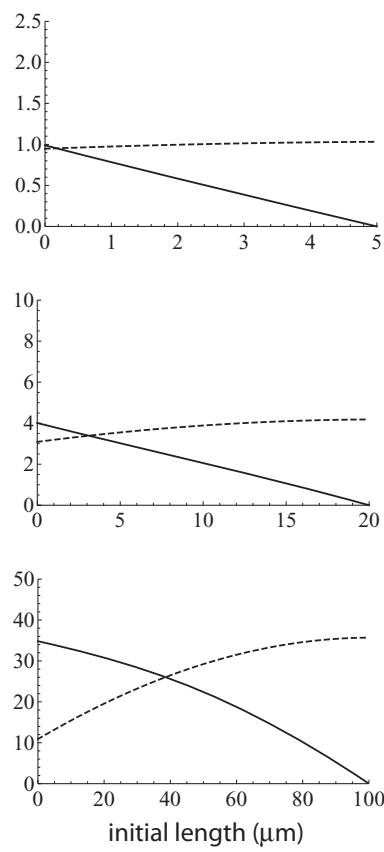

FIG. 4. Conditional MFPTs for reaching the barrier without shrinking to zero in between, $T_{A}^{B}(l,+)$ (solid line), and for shrinking to zero length without hitting the boundary in between, $T_{A}^{N}(l,+)$ (dashed line), as a function of the initial length $l$ for different values of the distance to the barrier $L$.

Finally, in Table II I give the MFPT $T_{\Omega_{L}}(0,+)$ for reaching the barrier starting from zero length in the growing state as calculated from Eq. (44).

I see that for a yeast MT, the largest boundary distance $L=100 \mu \mathrm{m}$ is effectively unbridgeable and that even the plant MT needs on average $\approx 10$ times its natural lifetime $\bar{t} \approx 20$ min to first reach the boundary although it is only $\approx 1.5$ times its natural length of $\bar{l} \approx 65 \mu \mathrm{m}$.

\section{THE GOPALAKRISHNAN-GOVINDAN SEARCH-AND-CAPTURE MODEL}

The first-passage-time model Gopalakrishnan and Govindan recently introduced [10] (hereafter referred to as GG) considers the problem of the "capture" of a chromosome by a MT "searching" for it. It has the following ingredients. The MT is nucleated at a rate $r_{n}$ from a centrosome in an arbitrary direction into a cone with a solid angle opening of $\Delta \Omega$. The centrosome is located at a distance $d$ from the chromosome, which has a cross-sectional area $a$, and, therefore, subtends a solid angle $\Delta \Omega_{c}=a / d^{2}$ as seen from

TABLE II. The MFPT $T_{\Omega_{L}}(0,+)$ (in min) as a function of the distance $L$.

\begin{tabular}{lcr}
\hline \hline$L(\mu \mathrm{m})$ & Yeast & Plant \\
\hline 5 & 8.89 & 3.29 \\
20 & 119.1 & 17.23 \\
100 & $2.9 \times 10^{6}$ & 266.55 \\
\hline \hline
\end{tabular}

the centrosome. The probability of being nucleated into a direction in which the target can possibly be hit is therefore given by $p_{c}=\Delta \Omega_{c} / \Delta \Omega$. When the MT is nucleated outside of the "target cone", it can potentially grow until it hits a cell boundary located at a distance I will call $D$ from the centrosome. At this boundary, the MT is initially stalled but experiences an increased catastrophe rate $r_{b}>r_{+}$.

I will now revisit this model, using the formalism derived in the previous sections. The state space of this model is conveniently represented by $\Omega=N \cup A_{b} \cup B \cup A_{c} \cup C$. Here, as before, $N$ is the nucleation state, $A_{b}$ are the active states with lengths in the interval $[0, D]$ in the directions that do not "see" the target, $B$ is the state of being at the cell boundary, $A_{c}$ are the active states with lengths in the interval $[0, d]$ and directions within the target cone, and, finally, $C$ is the state of being on the target chromosome. I nondimensionalize using the same prescription as in Sec. II C, denoting the additional parameters needed by $\Delta=D / \bar{l}$ and $\delta=d / \bar{l}$. Using the results of Sec. III A, I can immediately write down an expression for the search time starting from the nucleation state

$$
\begin{aligned}
T_{\Omega / C}(N)= & \frac{1}{\rho_{n}}+\left(1-p_{c}\right)\left\{T_{A_{b} \cup B}\left[(0,+)_{A_{b}}\right]+T_{\Omega / C}(N)\right\} \\
& +p_{c}\left(R_{A_{c}}^{C}\left[(0,+)_{A_{c}}\right] T_{A_{c}}^{C}\left[(0,+)_{A_{c}}\right]\right. \\
& \left.+R_{A_{c}}^{N}\left[(0,+)_{A_{c}}\right]\left\{T_{A_{c}}^{N}\left[(0,+)_{A_{c}}\right]+T_{\Omega / C}(N)\right\}\right) .
\end{aligned}
$$

The logic of this equation is simple. Starting from the nucleation state, the MT (on average) waits $1 / \rho_{n}$ before being nucleated. With probability $\left(1-p_{c}\right)$, the nucleation will be in a direction that cannot hit the target. In that case the MT will spend the origin-return time $T_{A_{b} \cup B}\left[(0,+)_{A_{b}}\right]$ in this part of state space before shrinking back to zero length and starting again from the nucleation state. With probability $p_{c}$, the initial nucleation is inside the target cone. In that case the MT either hits the target without first shrinking back to zero length with probability $R_{A_{c}}^{C}\left[(0,+)_{A_{c}}\right]$, taking time $T_{A_{c}}^{C}\left[(0,+)_{A_{c}}\right]$, or with probability $R_{A_{c}}^{N}\left[(0,+)_{A_{c}}=1-R_{A_{c}}^{C}\left[(0,+)_{A_{c}}\right.\right.$ shrinking back to zero length before hitting the target, taking time $T_{A_{c}}^{N}\left[(0,+)_{A_{c}}\right]$, and then trying again from the nucleation state. This process is illustrated in Fig. 5.

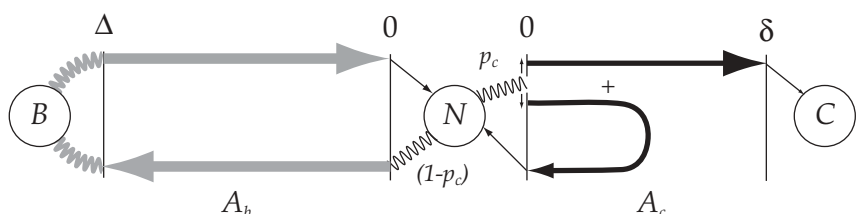

FIG. 5. Schematic illustration of the structure of the search process in the Gopalakrishnan-Govindan search-and-capture model. From the nucleation state the MT must either perform (with probability $1-p_{c}$ ) a fruitless search (gray arrows) in the directions in which it can interact with the cell boundary $A_{b}$, or (with probability $p_{c}$ ) it is nucleated in the right direction and then either directly traverses $A_{c}$ to reach the chromosome $C$ or shrinks back to zero length without reaching the target and must try again. 
Extracting $T_{\Omega / C}(N)$ from the relation (66) yields

$$
\begin{aligned}
p_{c} R_{A_{c}}^{C}\left[(0,+)_{A_{c}}\right] T_{\Omega / C}(N) \\
=\frac{1}{\rho_{+}}+\left(1-p_{c}\right) T_{A_{b} \cup B}\left[(0,+)_{A_{b}}\right] \\
\quad+p_{c}\left\{R_{A_{c}}^{C}\left[(0,+)_{A_{c}}\right] T_{A_{c}}^{C}\left[(0,+)_{A_{c}}\right]+R_{A_{c}}^{N}\left[(0,+)_{A_{c}}\right]\right. \\
\left.\quad \times T_{A_{c}}^{N}\left[(0,+)_{A_{c}}\right]\right\} .
\end{aligned}
$$

As I show in Appendix A, this is, apart from the changed notation, precisely the result derived by GG (see their Eq. (34) in Appendix A) from an explicit sum-over-histories argument. I also note the calculation of the fruitless search time, $T_{A_{b} \cup B}\left[(0,+)_{A_{b}}\right]$, in the directions not containing the target, is also readily simplified using the methods presented here:

$$
\begin{aligned}
T_{A_{b} \cup B}\left[(0,+)_{A_{b}}\right]= & R_{A_{b}}^{N}\left[(0,+)_{A_{b}}\right] T_{A_{b}}^{N}\left[(0,+)_{A_{b}}\right] \\
& +R_{A_{b}}^{B}\left[(0,+)_{A_{b}}\right]\left\{T_{A_{b}}^{B}\left[(0,+)_{A_{b}}\right]+\frac{1}{\rho_{b}}\right. \\
& \left.+T_{A_{b} \cup B}\left[(\Delta,-)_{A_{b}}\right]\right\}
\end{aligned}
$$

where in turn

$$
\begin{aligned}
T_{A_{b} \cup B}\left[(\Delta,-)_{A_{b}}\right]= & R_{A_{b}}^{N}\left[(\Delta,-)_{A_{b}}\right] T_{A_{b}}^{N}\left[(\Delta,-)_{A_{b}}\right] \\
& +R_{A_{b}}^{B}\left[(\Delta,-)_{A_{b}}\right]\left\{T_{A_{b}}^{B}\left[(\Delta,-)_{A_{b}}\right]+\frac{1}{\rho_{b}}\right. \\
& \left.+T_{A_{b} \cup B}\left[(\Delta,-)_{A_{b}}\right]\right\} .
\end{aligned}
$$

The latter expression allows $T_{A_{b} \cup B}\left[(\Delta,-)_{A_{b}}\right]$ to be expressed solely by splitting probabilities and conditional MFPTs. While GG use an ingenious symmetry argument interpreting a shrinking MT as a growing "anti"-MT to calculate these latter quantities, I point out that they can also be obtained in a straightforward manner from the differential equations presented in Secs. III B and III C.

\section{CONCLUSIONS AND OUTLOOK}

My aim was to present a structured approach to the problem of MTs interacting with boundaries. To this end, I relied exclusively on "backward" techniques, focussing on survival probabilities and their associated boundary value problems. The upshot of this approach is that it allows one to decompose a complex MFPT problem a priori into a closed form self-consistency problem involving a small set of relevant splitting probabilities and conditional MFPTs that readily follow from a proper disjoint decomposition of the state space. The utility of this approach is illustrated by its application to the Gopalakrishnan-Govindan model in which the key decomposition of the search time in terms of the time spent fruitlessly searching in the wrong directions, waiting in the nucleation state, and finally reaching the target is the starting point of the calculation rather than, as in Ref. [10], the result of collecting the results of intermediate steps in the calculation. I hope that the technique presented will serve as a convenient starting point for future applications to current problems in microtubule cytoskeleton organization.

\section{ACKNOWLEDGMENTS}

This work is part of the research program of the "Stichting voor Fundamenteel Onderzoek der Materie (FOM),"which is financially supported by the "Nederlandse Organisatie voor Wetenschappelijk Onderzoek (NWO)."

\section{APPENDIX A: EXPLICIT SOLUTIONS OF THE CONDITIONAL MFPTS}

In order to give a fairly concise expression for the conditional MFPTs, I need to introduce a number of convenient coefficients:

$$
\begin{gathered}
\alpha=\lambda_{+}-1, \\
\beta=\frac{1}{\lambda_{+} \lambda_{-}}\left(\frac{1}{\rho_{+}}+\frac{1}{\rho_{-}}\right), \\
\gamma=\frac{\lambda_{+}}{\rho_{-}}-\frac{\lambda_{-}}{\rho_{+}}, \\
\delta=\lambda_{+}\{\beta(1-\alpha)+\gamma\}, \\
\varepsilon=1+\alpha-\beta \rho_{+}+\alpha^{2} \beta \rho_{+}-\gamma \rho_{+}-\alpha \gamma \rho_{+} \\
=\lambda_{+}-\rho_{+} \delta .
\end{gathered}
$$

With these definitions, I find

$$
\begin{gathered}
T_{A}^{N}(\lambda,+)=\beta \lambda_{+}+\beta \lambda+\frac{1}{e^{\Lambda}-e^{\lambda}}\left\{-e^{\lambda}(2 \beta+\gamma)(\Lambda-\lambda)+R_{A}^{N}(\lambda,+)\left[\frac{1}{\rho_{+}}\left(e^{\Lambda}-1\right)+\delta-\alpha(2 \beta+\gamma) \Lambda\right]\right\} \\
T_{A}^{B}(\lambda,+)=\frac{1}{\rho_{+}} \frac{1}{e^{\lambda}+\alpha}\left\{\begin{array}{c}
\varepsilon+\alpha \rho_{+}(\beta+\gamma) \Lambda+\alpha \beta \rho_{+} \lambda+(\beta+\gamma) \rho_{+}(\Lambda-\lambda) e^{\lambda} \\
-R_{A}^{B}(\lambda,+)\left[\varepsilon+\alpha \rho_{+}(\beta+\gamma) \Lambda+\alpha \beta \rho_{+} \Lambda\right]
\end{array}\right\}
\end{gathered}
$$

In some cases I can use the known relations

$$
\begin{gathered}
1=\frac{1}{\lambda_{+}}-\frac{1}{\lambda_{-}}, \\
1=\frac{1}{\lambda_{+} \rho_{+}}+\frac{1}{\lambda_{-} \rho_{-}},
\end{gathered}
$$

to simplify even further. An example is the observation that

$$
\begin{aligned}
\beta \lambda_{+} & =\frac{1}{\lambda_{-}}\left(\frac{1}{\rho_{+}}+\frac{1}{\rho_{-}}\right)=\left(\frac{1}{\lambda_{+}}-1\right) \frac{1}{\rho_{+}}+\frac{1}{\lambda_{-} \rho_{-}} \\
& =1-\frac{1}{\rho_{+}} .
\end{aligned}
$$


TABLE III. Corresponding quantities between Ref. [10] and my own work.

\begin{tabular}{lcr}
\hline \hline GG & This work & Description \\
\hline$\Phi(d, T)$ & $\sigma_{A_{c}}^{C}\left(T \mid(0,+)_{A_{c}}\right)$ & Conditional waiting time distribution reaching the target without shrinking back to zero \\
$Q_{d}(T)$ & $\sigma_{A_{c}}^{N}\left(T \mid(0,+)_{A_{c}}\right)$ & Conditional waiting time distribution shrinking back to zero without reaching the target \\
$Q_{R}(T)$ & $\sigma_{A_{b}}^{N}\left(T \mid(0,+)_{A_{b}}\right)$ & Conditional waiting time distribution shrinking back to zero without reaching the boundary \\
$\Psi(T)$ & $\sigma_{A_{b} \cup B}\left(T \mid(0,+)_{A_{b}}, \exists \tau<T: \lambda_{\tau}=\Delta\right)$ & Waiting time distribution return to length zero after reaching the boundary at least once \\
\hline \hline
\end{tabular}

Taking the limit $\Lambda \rightarrow \infty$ then yields, as in this limit $R_{A}^{N}(\lambda,+)=1$,

$$
T_{A}^{N}(0,+)=1
$$

as claimed in the main text.

\section{APPENDIX B: FORMAL CORRESPONDENCE WITH THE GOPALAKRISHNAN- GOVINDAN MODEL}

Here, I provide the translation between the results of GG and my own by noting the following correspondences shown in Table III. From these correspondences I derive the identities

$$
\begin{gathered}
\tilde{\Phi}(d, 0)=\int_{0}^{\infty} d T \sigma_{A_{c}}^{C}\left(T \mid(0,+)_{A_{c}}\right)=R_{A_{c}}^{C}\left[(0,+)_{A_{c}}\right], \\
\tilde{\Phi}^{\prime}(d, 0)=-\int_{0}^{\infty} d T T \sigma_{A_{c}}^{C}\left(T \mid(0,+)_{A_{c}}\right) \\
=-R_{A_{c}}^{C}\left[(0,+)_{A_{c}}\right] T_{A_{c}}^{C}\left[(0,+)_{A_{c}}\right],
\end{gathered}
$$

$$
\begin{aligned}
& \tilde{Q}^{\prime}(d, 0)=-\int_{0}^{\infty} d T T \sigma_{A_{c}}^{N}\left(T \mid(0,+)_{A_{c}}\right) \\
& =-R_{A_{c}}^{N}\left[(0,+)_{A_{c}}\right] T_{A_{c}}^{N}\left[(0,+)_{A_{c}}\right], \\
& \tilde{Q}^{\prime}(R, 0)=-\int_{0}^{\infty} d T T \sigma_{A_{b}}^{N}\left(T \mid(0,+)_{A_{b}}\right) \\
& =-R_{A_{b}}^{N}\left[(0,+)_{A_{b}}\right] T_{A_{b}}^{N}\left[(0,+)_{A_{b}}\right], \\
& \Psi^{\prime}(0)=-\int_{0}^{\infty} d T T \sigma_{A_{b} \cup B}\left(T \mid(0,+)_{A_{b}}, \exists \tau<T: \lambda_{\tau}=\Delta\right) \\
& =-R_{A_{b}}^{B}\left[(\Delta,-)_{A_{b}}\right]\left\{T_{A_{b}}^{B}\left[(\Delta,-)_{A_{b}}\right]+\frac{1}{\rho_{b}}\right. \\
& \left.+T_{A_{b} \cup B}\left[(\Delta,-)_{A_{b}}\right]\right\} \text {. }
\end{aligned}
$$

$$
\begin{gathered}
T_{d}=-\frac{\tilde{\Phi}^{\prime}(d, 0)+\tilde{Q}^{\prime}(d, 0)}{\tilde{\Phi}(d, 0)}=\frac{R_{A_{c}}^{N}\left[(0,+)_{A_{c}}\right] T_{A_{c}}^{N}\left[(0,+)_{A_{c}}\right]+R_{A_{c}}^{C}\left[(0,+)_{A_{c}}\right] T_{A_{c}}^{C}\left[(0,+)_{A_{c}}\right]}{R_{A_{c}}^{C}\left[(0,+)_{A_{c}}\right]}, \\
T_{R}=-\frac{\tilde{Q}^{\prime}(R, 0)+\Psi^{\prime}(0)}{\tilde{\Phi}(d, 0)} \\
=\frac{R_{A_{b}}^{N}\left[(0,+)_{A_{b}}\right] T_{A_{b}}^{N}\left[(0,+)_{A_{b}}\right]+R_{A_{b}}^{B}\left[(\Delta,-)_{A_{b}}\right]\left\{T_{A_{b}}^{B}\left[(\Delta,-)_{A_{b}}\right]+\frac{1}{\rho_{b}}+T_{A_{b} \cup B}\left[(\Delta,-)_{A_{b}}\right]\right\}}{R_{A_{c}}^{C}\left[(0,+)_{A_{c}}\right]} \\
=\frac{T_{A_{b} \cup B}\left[(0,+)_{A_{b}}\right]}{R_{A_{c}}^{C}\left[(0,+)_{A_{c}}\right]}, \\
T_{v}=\frac{1}{\rho_{n} R_{A_{c}}^{C}\left[(0,+)_{A_{c}}\right]},
\end{gathered}
$$

so that, indeed, their expression

$$
\langle T\rangle=T_{d}+\frac{\left(1-p_{c}\right)}{p_{c}} T_{R}+\frac{1}{p_{c}} T_{v}
$$

fully coincides with Eq. (67).

[1] B. Alberts, A. Johnson, J. Lewis, M. Raff, K. Roberts, and P. Walter, Molecular Biology of the Cell, 4th ed. (Garland Science, New York, 2002).

[2] T. Mitchison and M. Kirschner, Nature (London) 312, 237 (1984).
[3] M. Dogterom and S. Leibler, Phys. Rev. Lett. 70, 1347 (1993).

[4] P. T. Tran, L. Marsh, V. Doye, S. Inoué, and F. Chang, J. Cell Biol. 153, 397 (2001).

[5] S. W. Grill, J. Howard, E. Schäffer, E. H. K. Stelzer, and A. A. Hyman, Science 301, 518 (2003). 
[6] C. Ambrose, J. F. Allard, E. N. Cytrynbaum, and G. O. Wasteneys, Nat. Commun. 2, 430 (2011).

[7] T. E. Holy and S. Leibler, Proc. Natl. Acad. Sci. USA 91, 5682 (1994).

[8] T. E. Holy, Ph.D. thesis, Princeton University, 1997 (unpublished).

[9] R. Wollman, E. N. Cytrynbaum, J. T. Jones, T. Meyer, J. M. Scholey, and A. Mogilner, Curr. Biol. 15, 828 (2005).

[10] M. Gopalakrishnan and B. S. Govindan, Bull. Math. Biol. 73, 2483 (2011).

[11] S. Redner, A Guide to First-Passage Processes (Cambridge University Press, Cambridge, 2001).

[12] M. E. Janson, M. E. de Dood, and M. Dogterom, J. Cell Biol. 161, 1029 (2003).
[13] M. Dogterom, J. W. J. Kerssemakers, G. Romet-Lemonne, and M. E. Janson, Curr. Opin. Cell Biol. 17, 67 (2005).

[14] B. Govindan and W. Spillman, Phys. Rev. E 70, 032901 (2004).

[15] R. J. Rubin, Proc. Natl. Acad. Sci. 85, 446 (1988).

[16] D. Williams, Diffusions, Markov Processes, and Martingales Volume I: Foundations (John Wiley \& Sons, Chichester, 1979).

[17] D. J. Bicout, Phys. Rev. E 56, 6656 (1997).

[18] M. E. Janson, R. Loughlin, I. Loïodice, C. Fu, D. Brunner, F. J. Nédélec, and P. T. Tran, Cell (Cambridge, MA, US) 128, 357 (2007).

[19] J. W. Vos, M. Dogterom, and A. M. C. Emons, Cell Motil. Cytoskeleton 57, 246 (2004). 